\title{
Filling of Traps with Electrons in Insulators Subjected to Intense Electron Irradiation
}

\author{
V.D. Kulikov \\ Tomsk Agriculture Institute, 19, K. Marks str., Tomsk, 634009, Russia \\ E-mail:vdkulikov@rambler.ru
}

\begin{abstract}
The efficiency of nonequilibrium electron trapping by capture centers in alkali halide crystals, quartz, and polymethyl-methacrylate exposed to an intense electron beam with a beam current density of about $20 \mathrm{~A} / \mathrm{cm}^{2}$ is studied. The trapped charge is estimated from the amount of irradiation-induced electrification of high-resistivity materials. It is shown that traps having captured thermalized electrons become depleted via impact ionization due to the primary electrons of the beam and secondary electrons.
\end{abstract}

\section{Introduction}

Capture of nonequilibrium electrons and holes is a central process in radiation-induced electrification, radiation-induced conductivity, and chemiluminescence. Carrier capture becomes of special importance in experiments with intense charged particle beams, which generate additional traps and make uncertain a relationship between the concentrations of trapped and free carriers.

A relationship between the concentrations of trapped and free carriers can be derived from the electrification of an insulator by trapped and free thermalized electrons of the beam. In turn, discrimination between the trapped and free electrons of the beam allows the calculation of free charge accumulation in a trap-free material [1, 2].

In this work, we study the efficiency of nonequilibrium electron trapping (localization) by capture centers in insulators exposed to intense pulsed electron irradiation. The trapped charge was estimated by comparing experimental and analytical dependences of the material electrification on the electron beam current density.

\section{Radiation-induced charge accumulation in insulators}

The scheme used in experiments on electron-beaminduced electrification of insulators is shown in $[1,2]$. The samples, plates measuring $40 \times 40(0.3-1.0) \mathrm{mm}$, were made of pure $\mathrm{KCl}, \mathrm{NaCl}$, and $\mathrm{LiF}$ single crystals; fused and crystalline quartz; and polymethyl-methacrylate. The electron beam had the following parameters: a mean energy of accelerated electrons of $0.25 \mathrm{MeV}$, FWHM of $18 \mathrm{~ns}$, and current density $I$ ranging from 4 to $30 \mathrm{~A} / \mathrm{cm}^{2}$.
In the method used in this work, the waveform of the electric field strength beyond the electron range, $E_{2}^{\prime}(t)$, is obtained by integrating displacement current density over time.

\section{Results and discussion}

The dependence of the peak strength of the electric field value of $E_{2}^{\prime}$ on the beam current density for different insulators is illustrated in Fig. 1.

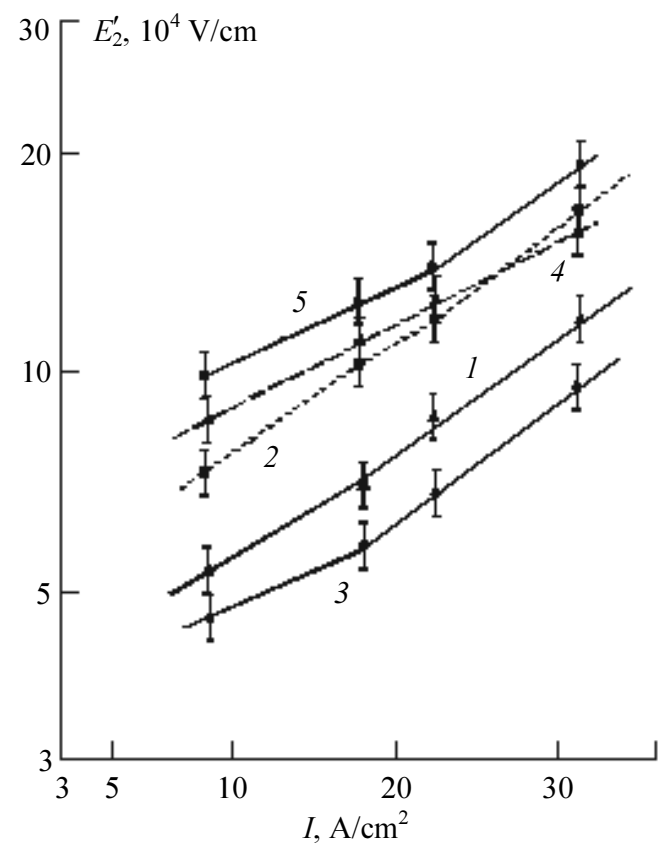

Fig. 1. Peak strength of the electric field vs. beam current density $I$ for $\mathrm{NaCl}(0.75)(1), \mathrm{KCl}(0.50)(2), \mathrm{LiF}(0.75)$ (3), $\mathrm{SiO}_{2}$ (0.87) (4), and polymethyl-methacrylate (1.0) (5). The parenthesized figures are sample thicknesses in millimeters

The value of depends on the beam current density, sample thickness, and bulk resistivity. In the $\mathrm{KCl}$ samples, is 1.8 times higher than in $\mathrm{NaCl}$ for close values of the radiation-induced conductivity [7]. According to [2], this difference is approximated well by the relationship $\left(x_{3 \mathrm{NaCl}}-x_{2}\right) /\left(x_{3 \mathrm{KCl}}-x_{2}\right) \approx 2$.

When the radiation-induced conductivity varies with the beam current density as $\sigma(t) \sim I^{0.5}$ and electron trapping is absent, the field strength is bound to vary as $I^{0.5}$, according to [2]. The $\mathrm{NaCl}, \mathrm{KCl}$, and $\mathrm{LiF}$ samples meet such a dependence, starting from low $\left(8.8 \mathrm{~A} / \mathrm{cm}^{2}\right)$ beam current densities. For fused quarz 
and polymethylmethacrylate, the field strength varies in a more complicated manner. In the current density range from 4.4 to $12.0 \mathrm{~A} / \mathrm{cm}^{2}$, the field strength is proportional to $I^{0.4}$ and tends toward the dependence $\sim I^{0.5}$ at fields higher than $22.5 \mathrm{~A} / \mathrm{cm}^{2}$.

Such a pattern of the insulator electrification indicates that the trapping of electrons decreases with increasing beam current density. Of interest are therefore the mechanism of electron trap depletion and the manifestation of this mechanism in radiation-induced conduction and defect formation.

In general, carrier traps in an insulator are structural defects and centers produced by ionizing radiation.

It was conjectured [3] that the concentration of Schottky defects in alkali halide crystals does not exceed $10^{13} \mathrm{~cm}^{-3}$. In glasses, the electron state density functions at the edges of allowed bands exhibit tails resulting from internal field density (strength) fluctuations. Since the spatial extension of the tails is much greater than the atomic spacing, a quasi-continuous, rather than discrete, spectrum of electron states arises with a modulation depth of 0.2-0.4 eV [4].

Ionizing radiation generates nonequilibrium electrons and states in alkali halide crystals [3,5]. The basic channel of nonequilibrium electron capture is trapping by $F$ centers with the formation of $F$ centers.

It may be supposed that the depletion of structural defects and $F$ centers is associated with the influence of an electric field. It was noticed that, when a high electric field $\left(10^{4}-10^{5} \mathrm{~V} / \mathrm{cm}\right)$ is applied to alkali halide crystals, glasses, or crystalline quartz excited by pulsed X-ray radiation, the radiation-induced conductivity increases by a factor of 1.5-2.0 [1, 6-9]. It was shown $[1,8,9]$ that the conductivity law in an electric field corresponds to trap depletion due to impact ionization by conduction electrons accelerated in the electric field. The energy of activation of capture centers in $\mathrm{KCl}, \mathrm{NaCl}, \mathrm{CsI}, \mathrm{KBr}$, and $\mathrm{KI}$ crystals falls into the interval $0.03-0.05 \mathrm{eV}$ and their concentration is on the order of $10^{14} \mathrm{~cm}^{-3}$. For example, the concentration of $F$ centers in $\mathrm{KBr}$ crystals excited by x-ray radiation is about $3 \cdot 10^{14} \mathrm{~cm}^{-3}$. However, the energy of activation of traps mentioned above is much lower than the energy of ionization of $F$ and $F$ centers from both the ground and excited states $(1.5-2.0$ and $\approx 0.1 \mathrm{eV}$, respectively) [10].

It seems most likely that electron traps observed in alkali halide crystals are divacancies formed by nearby cation and anion vacancies [1]. When a CsI crystal was heated to $600{ }^{\circ} \mathrm{C}$ in air for $1 \mathrm{~h}$ and then quenched to room temperature, the absorption in the $\alpha$ band (the absorption band of halogen ions that lies near the absorption band of an anion vacancy [3]) was found to increase by a factor of 1.2 and the conduction current density decrease roughly threefold. This can be explained by the separation of divacancies and capture of free carriers by anion vacancies with the formation of $F$ centers. Heating of the $\mathrm{KBr}$ sample to $600{ }^{\circ} \mathrm{C}$ for
$3 \mathrm{~h}$ changed neither the absorption in the $\alpha$. band nor the conductivity [1], which is explained by a higher energy of activation of anion vacancy migration in $\operatorname{KBr}(0.92$ versus $0.39 \mathrm{eV}$ in CsI [3]).

In crystalline quartz and K208 glass, electrons are likely to be captured by the tails of the electron state density function. The basic reason for modulation is density fluctuations due to a variable length of chemical bonds [4]. According to $[1,8]$, the activation energy required for the $\mathrm{I}-\mathrm{V}$ characteristic to become ohmic is $\approx 0.1 \mathrm{eV}$ for crystalline quartz and $\approx 0.2 \mathrm{eV}$ for K208 glass.

When the alkali halide crystals are excited by nanosecond and picosecond electron pulses with a current density of 1-2 [6] and $30 \mathrm{~A} / \mathrm{cm}^{2}$ [7], respectively, the I-V curves are linear. It seems that the acting mechanism of electron transfer to the conduction band is independent of the electric field with a strength of $10^{4}-10^{5} \mathrm{~V} / \mathrm{cm}$.

The defect formation in alkali halide crystals irradiated by a nanosecond electron pulse (with the same parameters as above) is described in [11]. At the nitrogen point, short-lived $F$ centers (with a lifetime comparable to the electron excitation time) and stable $F$ centers related to the presence of spatially separated $F$ and $H$ pairs are generated. At room temperature, the latter prevail and their yield under electron beam irradiation is fairly high: 0.1 per electron-hole pair according to optical absorption data (the number of electron-hole pairs equals the electron pulse energy divided by $1.5 W_{g}$, where $W_{g}$ is the energy gap of the crystal). In the $\mathrm{KCl}$ crystal exposed to an electron pulse with a current density of $5 \mathrm{~A} / \mathrm{cm}^{2}$, the concentration of $F$ centers was about $5 \cdot 10^{16} \mathrm{~cm}^{-3}$.

It is essential that the yield of short-lived $F$ centers, $\eta_{F}$, and self-localized excitons, $\eta_{e}$, decreases with increasing beam current density (Fig. 2), as was also shown in [11].

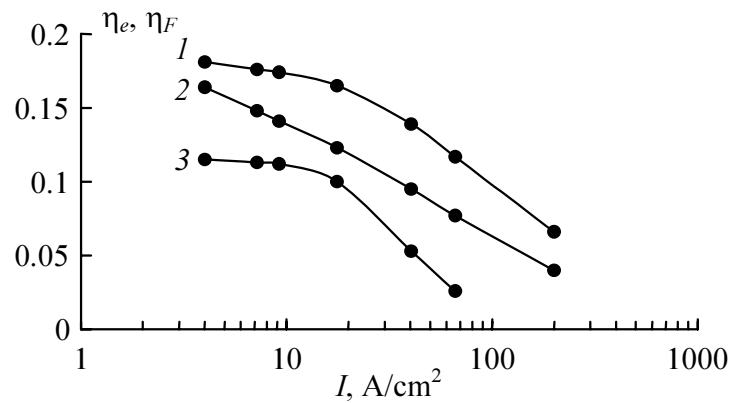

Fig. 2. Yield of self-localized excitons, $\eta_{e}$, in $\operatorname{CsBr}(l)$ and $\mathrm{KI}(2)$ and of short-lived $F$ centers, $\eta_{F}$, in $\mathrm{KCl}$ vs (3). Beam current density $I$ at $80 \mathrm{~K}$ [11]

This decrease is the most significant (by three to four times) in the beam current density range from 20 to $200 \mathrm{~A} / \mathrm{cm}^{2}$. For self-localized excitons, $\eta_{e}$ varies with $I$ as $\sim I^{-0.79}$ in the $\mathrm{CsBr}$ crystal (curve 1 ) and as $\sim I^{-0.78}$ in the KI crystal (curve 2); yield $\eta_{F}$ of shortlived $F$ centers in the $\mathrm{KCl}$ crystal varies with $I$ as 
$\eta_{\mathrm{F}} \sim I^{-1.1}$ (curve 3 ). In $\mathrm{KCl}$, the yield of stable $F$ centers increases by a factor of 2.3 (from 0.012 to 0.027 ) in the beam current density range $10-60 \mathrm{~A} / \mathrm{cm}^{2}$; in $\mathrm{CsBr}$, it changes insignificantly (from 0.014 to 0.015 ) in the range $10-200 \mathrm{~A} / \mathrm{cm}^{2}$. Thus, the total concentration of short-lived $F$ centers and self-localized excitons, which is proportional to $-\eta I$, varies with current density in the range $20-200 \mathrm{~A} / \mathrm{cm}^{2}$ only slightly.

The authors of [11] believe that the drastic decrease in the yield of relaxed self-localized excitons and shortlived $F$ centers in alkali halide crystals with increasing excitation (current) density is associated with the fact that some of the electrons are captured by $V_{k}$ centers in the vibrationally excided state rather than by thermalized $V_{k}$ centers. The presence of excitons with excess starting vibrational energy apparently raises the probability of nonradiative decays giving rise to stable $F$ centers compared with the relaxation probability of selflocalized electrons.

In fused and crystalline quartz, short-lived $F$ centers are effectively generated under the action of ionizing radiation; however, the radiation-induced accumulation of $F$ centers is minor because of a high rate of defect annihilation [3, 12].

\section{Trap depletion model}

It is the author's opinion that, when insulators are exposed to intense electron irradiation, the electrons localized by structural defects and $F$ centers are released through impact ionization by beam electrons and secondary electrons with energy comparable to the energy gap of the insulators.

The decay of short-lived $F$ centers and selflocalized excitons considered above (Fig. 2) can be attributed to their ionization by secondary electrons. It appears that, at $295 \mathrm{~K}$ too, stable $F$ centers decay exactly at the instant of irradiation and then their concentration (corresponding to $20 \mathrm{~A} / \mathrm{cm}^{2}$ ) varies with beam current density only slightly. Trap ionization contributes to the phenomenon of radiation-induced conduction through the release of localized charge carriers.

One can show that the occupancy of traps decreases with increasing beam current density. During irradiation, traps with concentration $N_{t}$ capture both primary and thermalized secondary electrons. The concentration of secondary electrons exceeds that of primary ones by three to four orders of magnitude, but the capture of secondary electrons does not violate the electroneutrality of the sample. The concentration of thermalized secondary electrons can be represented as the product $G_{e} \tau_{0}$, where $G_{e}$ is the volume rate of carrier generation and $\tau_{0}$ is the lifetime of a thermalized electron. Filled traps are ionized by secondary electrons at the stage of thermalization. Their concentration is $G_{e} \tau_{1}$, where $\tau_{1}$ is the thermalization time. The kinetic equation for trapped electron concentration $\Delta n$ has the form

$$
\frac{\partial \Delta n}{\partial t}=\left(N_{t}-\Delta n\right) G_{e} \tau_{0} v_{T} s_{0}-\Delta n G_{e} \tau_{1} v_{1} s_{1}
$$

Here $\left(N_{t}-\Delta n\right)$ is the density of empty traps; $v_{1}$ is the thermal velocity of electrons at the stage of thermalization; and $s_{0}$ and $s_{1}$ are the cross sections of capture and trap ionization, respectively. The first term on the right of (1) is responsible for trap filling, and the second one stands for trap depletion. In the quasistationary case, we have

$$
\Delta n=\frac{N_{t} \tau_{0} v_{T} s_{0}}{\tau_{1} v_{1} s_{1}\left(\tau_{0} v_{T} s_{0} / \tau_{1} v_{1} s_{1}+1\right)} .
$$

In alkali halide crystals, the mean energy of thermalized electrons at temperatures close to $300 \mathrm{~K}$ is about $0.02 \mathrm{eV}$ and the energy of secondary electrons at the stage of generation is $\approx 6 \mathrm{eV}$. Taking into account that the thermal velocity of electrons varies as the square root of their energy, we get $v_{T} / v_{1} \approx 0.06$. The electron thermalization time in the conduction band of an insulator is $\tau_{1} \sim 10^{-12} \mathrm{~s}$ [13], and the electron lifetime in the conduction band of $\mathrm{KCl}$ excited by an electron beam with a current density of $100 \mathrm{~A} / \mathrm{cm}^{2}$ is $\tau_{0} \sim 10^{-11} \mathrm{~s}$ [7]. Since electrons are captured, as a rule, by neutral centers and ionization is related to a charged center, we have $s_{0} / s_{1} \leqslant 0.1 \quad[13]$; hence, $\Delta n \approx 0.04 N_{t}$. As the beam current density grows, electron thermalization time $\tau_{1}$ in the conduction band of an insulator remains unchanged; however, the trapped charge decreases, because so does the lifetime of thermalized carriers. Assuming that the beam current density is $300 \mathrm{~A} / \mathrm{cm}^{2}$ and taking into account electronhole quadratic recombination, $\tau_{0} \approx I^{-0.5}$, we find that $\tau_{0} \approx 0.6 \cdot 10^{-11} \mathrm{~s}$ and $\Delta n \approx 0.028 N_{t}$. With a rise in the bean current density, $\Delta n$ will decrease in proportion to $I^{-0.5}$.

If the field strength in the insulator (Fig. 1) is represented as a sum of two components (one related to free charge accumulation and the other to the trapped charge) and it is assumed that the amount of the trapped charge at $22.5 \mathrm{~A} / \mathrm{cm}^{2}$ can be neglected, the trapped-to-free charge ratio at $4.4 \mathrm{~A} / \mathrm{cm}^{2}$ will be 0.13 in $\mathrm{LiF}$ and 0.3 in fused quartz and polymethylmethacrylate.

\section{Conclusion}

It is shown that irradiation of alkali halide crystals, quartz, and polymethylmethacrylate by an intense pulsed electron beam causes an effective depletion of centers having captured thermalized electrons. A trap depletion model is suggested that implies impact ionization by the primary electrons of the bean and secondary electrons. The trap depletion and decay of $F$ centers lead one to inspect radiation-induced electrification and radiation-induced conduction for the behavior of thermalized beam electrons and secondary electrons in the conduction band of an insulator. 
A considerable ionization of $F$ centers and selflocalized excitons may appreciably influence the light absorption and luminescence intensity in optical materials subjected to a powerful electron beam.

\section{References}

[1] V.D. Kulikov, Electrification of High-Resistance Materials in High Fields of Pulsed Radiation, Tomsk, TGU, 2004.

[2] V.D. Kulikov, Zh. Tekh. Fiz. 77/5, 23 (2007).

[3] Ch.B. Lushchik and A.Ch. Lushchik, Decay of Electron Excitations with Generation of Defects in Solids, Moscow, Nauka, 1989.

[4] A.R. Silin and Yu.R. Zakis, Izv. Akad. Nauk Latvii, Ser. Phis. Tekh. Nauk 5, 68 (1987).

[5] H. Fujiwara, T. Susuki, and K. Tanimura, J. Phys. Condens. Matter 9, 923 (1997).
[6] D.I. Vaisburd, High-Energy Solid-State Electronics, Novosibirsk, Nauka, 1982.

[7] B.P. Aduev, V.N. Shvaoeko, and V.M. Fomchenko, Izv. Tomsk. Politekh. Univ. 303 (2), 26 (2000).

[8] V.D. Kulikov and Yu.V. Lisyuk, Zh. Tekh. Fiz. 9, 51 (2000) [Tech. Phys. 45, 1141 (2000)].

[9] V.D. Kulikov, Izv. Vyssh. Uchebn. Zaved., Fiz. 7, 62 (2001).

[10] I.A. Parfianovich and E.E. Penzina, Electron Dye Centers in Ionic Crystals, Irkutsk, IGU, 1977.

[11] V.M. Lisitsyn, V.I. Korepanov, and V.Yu. Yakovlev, Izv. Vyssh. Uchebn. Zaved., Fiz. 11, 5 (1996).

[12] B.P. Gritsenko, V.M. Lisitsyn, and V.M. Stepanchuk, Fiz. Tverd. Tela 23, 393 (1981) [Sov. Phys. Solid State 23, 222 (1981)].

[13] M.A. Elango, Elementary Inelastic Radiation Processes, Moscow, Nauka, 1988 (New York, AIP, 1993). 